\title{
Semiempirical zero-dimensional approach for the estimation of beam features from plasma parameters in proton sources
}

\author{
G. Castro 10 \\ INFN Laboratori Nazionali del Sud, via S. Sofia 62, 95123 Catania, Italy
}

(Received 3 June 2020; accepted 9 September 2020; published 28 September 2020)

\begin{abstract}
Beam intensity, proton fraction, and beam emittance represent the most important characteristics of a beam extracted by a proton source. Beam parameters are strongly related to the parameters of the plasma from which the beam is extracted. The recent improvement of plasma diagnostics devoted to compact ion sources, and, in particular, the development of optical emission spectroscopy, allows an ever more reliable measurement of plasma parameters, also during source operations. In this paper, a zero-dimensional approach able to estimate the beam intensity and the ion fraction from plasma parameters in a proton source is introduced. The model is based on a simplified solution of the balance equation system in a hydrogen plasma achieved by including the electron density, the electron temperature, and the relative neutral abundance ratio $\mathrm{H} / \mathrm{H}_{2}$ within the equations. This approach permits one to reduce the balance equation system to four equations in four unknowns, namely, the $\mathrm{H}^{+}, \mathrm{H}_{2}^{+}$, and $\mathrm{H}_{3}^{+}$density and the confinement time. The balance equation system is solved by means of a homemade numerical code. The model has been applied to the beam generated by the Proton Source for the European Spallation Source (PS-ESS), during its commissioning at Istituto Nazionale di Fisica Nucleare, Laboratori Nazionali del Sud in Catania. The extracted current and ion fraction estimated by this approach have been compared with the experimental results obtained by the PS-ESS beam diagnostics. The proton confinement time estimated by this approach has been compared with the results of a theoretical model based on a diffusion model. The comparison demonstrates that the model has achieved a surprising capability of estimation. The paper discusses the main characteristics of the zero-dimensional approach together with its main advantages and limits. The perspectives for further improvement of the model are also discussed.
\end{abstract}

DOI: 10.1103/PhysRevAccelBeams.23.093402

\section{INTRODUCTION}

Microwave discharge ion sources (MDIS) and electron cyclotron resonance ion sources (ECRIS) are currently the most used devices to feed particle accelerators because of their compactness and reliability [1]. MDIS are usually used for the generation of protons, $\mathrm{H}_{2}^{+}$, and light ion beams characterized by low emittance, high stability, and beam intensity up to $100 \mathrm{~mA}[2-6]$.

To be able to improve the ion source performances, it is of great value to have an in-depth understanding of the mechanism occurring in the plasma. This can be achieved by the implementation of diagnostic techniques, such as Langmuir probe diagnostics, x-ray diagnostics, interferopolarimetry, and optical emission spectroscopy (OES) [7]. Among them, OES is probably the most remarkable for application in working ECRIS and MDIS: It requires very

Published by the American Physical Society under the terms of the Creative Commons Attribution 4.0 International license. Further distribution of this work must maintain attribution to the author(s) and the published article's title, journal citation, and DOI. little room for operation (just a quartz window to access the plasma light), it does not directly affect nor is affected by plasma (although indirect effects due to the optical windows can arise), and it operates also in high-voltage conditions, i.e., the usual working conditions in ECRIS and MDIS.

According to the sensitivity of the instrumentation, OES allows one to evaluate the plasma emission lines that, analyzed with a proper collisional radiative model, enable achieving the electron density $n_{e}$, the electron temperature $T_{e}$ of the cold electron population $(0-100 \mathrm{eV})[8,9]$, the ion temperature $T_{i}$ [10], the neutral relative abundances, the rotovibrational molecular spectrum [11], and the concentration of highly charged ions in plasmas by acquiring the electromagnetic spectrum emitted by the plasma in the optical domain. Figure 1 shows a typical spectrum acquired in a MDIS ion source: Balmer lines and a Fulcher band are evidenced.

More information on the OES technique and its applications can be found in Refs. [12,13].

The goal of this paper is to introduce a zero-dimensional semiempirical approach able to relate univocally the beam parameters to the plasma parameters in proton sources and, 


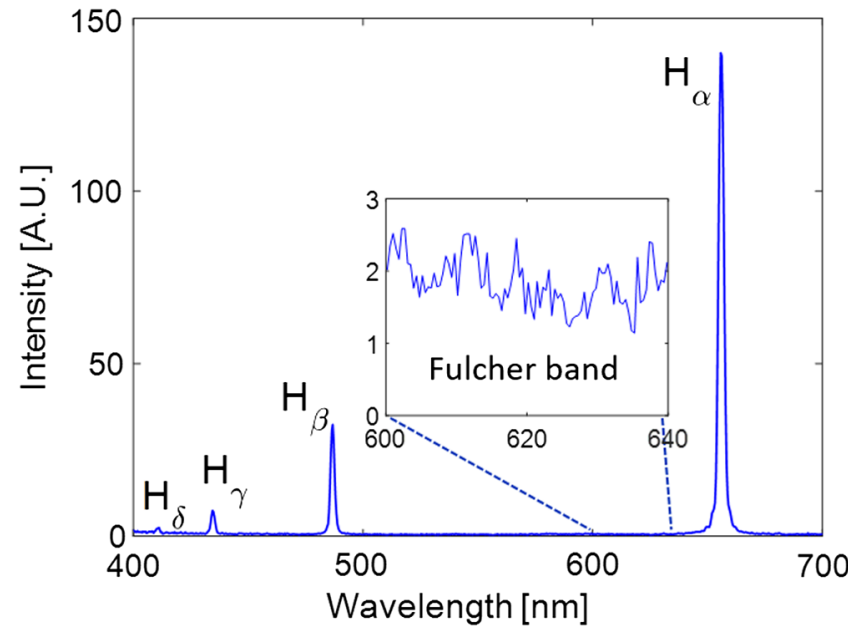

FIG. 1. Example of hydrogen spectrum used for achieving the PS-ESS plasma parameters.

therefore, to permit the real-time estimation of the beam parameters from plasma parameters. In past decades, numerical simulations have already become a valuable tool for improving the understanding of discharge physics and can provide theoretical predictions, especially in cases where diagnostic devices are expensive or difficult to include in an experimental setup. Zero-dimensional models applied to hydrogen discharges have been already reported [14-20]. Four fundamental conservation laws are usually used in the formulation of a zero-dimensional mode: (i) mass conservation, described via a particle balance for each species except for the $\mathrm{H}_{2}$ molecules; (ii) charge conservation, reduced to the quasineutrality condition; (iii) neutral density conservation, determined by the equation of state for the total particle number density; and (iv) the energy conservation law, expressed through power balance. The resulting balance conditions consist of a system of 24 coupled nonlinear ordinary differential equations which can be numerically solved to obtain relevant plasma parameters [14].

This paper simplifies the aforementioned balance equation system by including the plasma parameters evaluated by OES diagnostics and the neutral pressure as input parameters. This approach has allowed estimation of the in-plasma density of $\mathrm{H}^{+}, \mathrm{H}_{2}^{+}$, and $\mathrm{H}_{3}^{+}$ions. The classical Child-Langmuir law conditions allow one to estimate the concentration of $\mathrm{H}^{+}, \mathrm{H}_{2}^{+}$, and $\mathrm{H}_{3}^{+}$ions in the beam extracted from and the total current [21].

To validate this semiempirical approach, the MDIS proton source for the European Spallation Source (PSESS) has been used as a test bench [22]. PS-ESS was designed, built, and commissioned in the framework of the ESS project at the Istituto Nazionale di Fisica Nucleare, Laboratori Nazionali del Sud (INFN-LNS) to become the injector of the ESS linac. PS-ESS works in pulsed waves (6 ms pulse length, $14 \mathrm{~Hz}$ repetition rate) and produces more than $100 \mathrm{~mA}$ of proton current with an emittance lower than $0.2 \pi \cdot \mathrm{mm} \cdot \mathrm{mrad}$, according to the ESS project requirements. More information about PS-ESS and its characteristics can be found in Refs. [23,24].

During the commissioning phase, four diagnostics have been used to characterize the beam extracted by PS-ESS. In particular, we measured (i) the total current from a highvoltage power supply by a wide-bandwidth ac current transformer (ACCT); (ii) the species fraction in the lowenergy beam transport (LEBT) by a Doppler-shift measurement unit; (iii) the beam intensity at the end of the LEBT by a Faraday cup; and (iv) the beam emittance by an emittance measurement unit.

Measurements of PS-ESS plasma parameters by OES have been also performed. The electron density $n_{e}$, the electron temperature $T_{e}$, and the neutral relative abundances $N_{\mathrm{H}} / N_{\mathrm{H}_{2}}\left(N_{\mathrm{H}}\right.$ and $N_{\mathrm{H}_{2}}$ being the density of atomic hydrogen $\mathrm{H}$ and molecular hydrogen $\mathrm{H}_{2}$, respectively) have been estimated by comparing the differences between experimental and theoretical line ratios (Yacora package [25]) of Balmer lines and the Fulcher band for any operative configuration. The statistical error in the minimization procedure allows one to evaluate the plasma parameter error (see Ref. [26] for further information).

A comparison between beam parameters estimated by the semiempirical approach and experimental data acquired in the same experimental conditions has been used to validate the approach. Either the extracted current or the ion fractions are predicted with accuracy. Moreover, the confinement time estimated by the semiempirical approach has been compared with a theoretical diffusion model in MDIS. Plasma parameters used as input parameters in this work are available in Ref. [26].

\section{HYPOTHESIS AND ASSUMPTIONS}

The semiempirical approach presented in this work connects the plasma parameters $\left(n_{e}, T_{e}\right.$, and $\left.N_{\mathrm{H}} / N_{\mathrm{H}_{2}}\right)$ to the beam parameters under a number of hypotheses, approximations, and assumptions regarding the beam generation from plasma and the characteristics of the plasma parameters evaluated by OES that will be discussed in this section.

\section{A. Assumptions concerning the beam generation from plasma}

Generation and extraction of an ion beam from ECRIS plasma is a topic discussed for several decades. In this work, a series of reasonable approximations have been introduced to simplify the physical problem: (1) Ions move along a flux tube parallel to the magnetic field with a radius equal to the extraction hole $r$ (see Fig. 2). This hypothesis is based on the consideration that, in MDIS, the parallel diffusion coefficient $D_{\|}$is much larger than the radial diffusion coefficient $D_{\perp}$. Therefore, plasma can be 


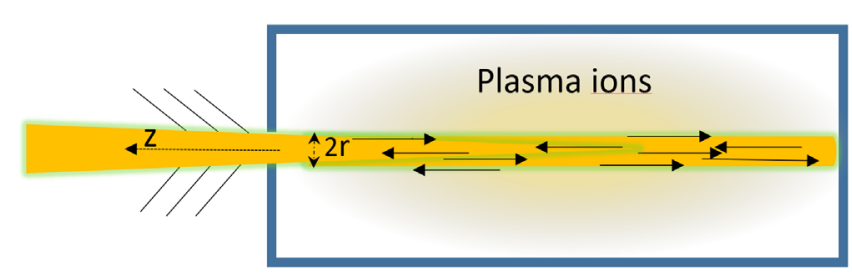

FIG. 2. Schematic view of a magnetic flux tube in a proton source. Because of the negligible radial diffusion, ions move along a magnetic tube flux tube toward the extraction hole [27].

considered frozen to the magnetic field lines [27-29]. (2) Ion motion is isotropic along the axial direction. Ions have equal probability to move toward the extraction hole or toward the opposite direction. Therefore, just one-half of the ions in the magnetic flux tube move toward extraction. (3) All ions in the flux tube approaching the plasma meniscus are extracted. (4) Ions enter the extraction region with Bohm velocity $v_{\text {Bohm }}$ [28]. This is the velocity with which ions enter the plasma sheath. $v_{\mathrm{Bohm}}^{i}$ depends on the electron temperature $T_{e}$ and ion temperature $T_{i}$ (measured in $\mathrm{eV}$ ) and on ion mass $M_{i}$ as follows:

$$
v_{\mathrm{Bohm}}^{i}=\sqrt{\frac{e\left(T_{i}+T_{e}\right)}{M_{i}}} .
$$

(5) The Child-Langmuir hypothesis is assumed: During extraction, the beam current density $J_{i}(z)$ (where the subscript $i$ represents each one of the three species that constitute the extracted beam, i.e., $\mathrm{H}^{+}, \mathrm{H}_{2}^{+}$, and $\mathrm{H}_{3}^{+}$, and $z$ is the distance from the extraction sheath) does not depend on the position $z$ in the gap [21]. Current density conservation implies that $J_{i}(z)$ can be evaluated in any point of the gap and, in particular, at the position $z=0$. It follows that

$$
J_{i}(z)=J_{i}(z=0)=q n_{i} v_{\text {Bohm }}^{i} .
$$

This strategy allows one to avoid any consideration about the influence of the geometry of the extraction system. In particular, one can assume the current value supplied by the ACCT as the total extracted beam current. It is well known that the ACCT current is due to two contributions, the positive ion current exiting the ion source and the electron current (generated by residual gas ionization in the LEBT) entering the source. However, the second contribution is generally much smaller than the first one, and, for the scope of this work, it can be neglected.

\section{B. Assumptions concerning plasma parameters}

The mode operation of PS-ESS and the experimental approach used to measure the plasma parameters imply the use of further approximations and hypothesis concerning plasma characteristics: (1) Plasma parameters are assumed to not change considerably with the chamber radius.

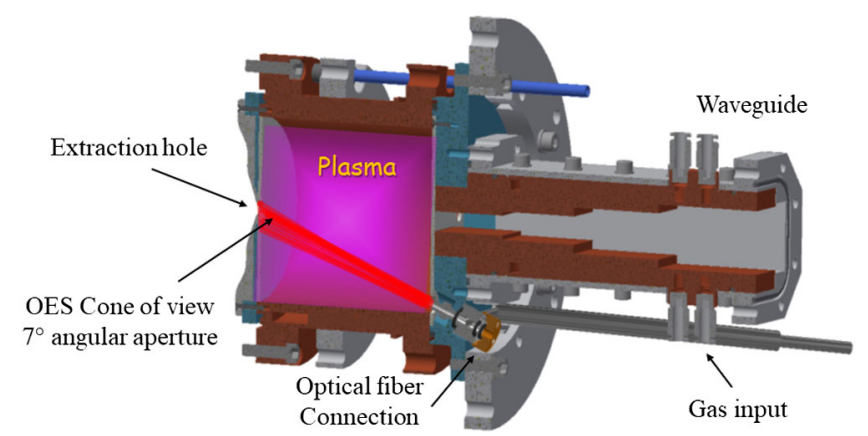

FIG. 3. Sketch of the OES experimental setup including the arrangement of the diagnostics.

Indeed, the first hypothesis concerning the beam generation requires plasma parameters to be ideally evaluated along the magnetic flux tube from which ions are extracted. In real experiments, this measurement cannot be performed because of mechanical constraints. In the case of the PS-ESS test bench [26], OES plasma parameters have been evaluated by means of an opportune optical fiber connection to the gas input. This has implied a determination of plasma parameters from an oblique cone of view intercepting the extraction hole, as shown in Fig. 3. The hypothesis of small dependence of plasma parameters from the radius allows considering OES measurements as representative of a measurement performed along the magnetic flux tube. (2) Although PS-ESS is operated in a $6 \mathrm{~ms}$ pulsed wave, we suppose that the variation of plasma parameters during plasma turn on and off phases is negligible. Figure 4 shows the $6 \mathrm{~ms}$ beam pulse measured by the ACCT for values of microwave power increasing from 100 to $1200 \mathrm{~W}$ through $10 \mathrm{~W}$ steps. Extracted current is almost constant in the temporal interval $0.4-6 \mathrm{~ms}$ for

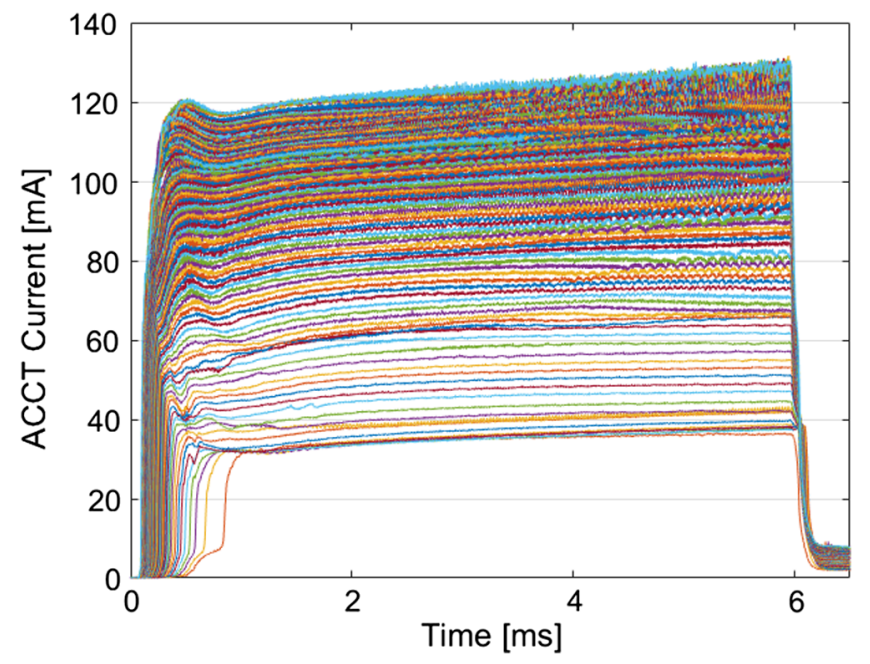

FIG. 4. PS-ESS beam pulse measured by the ACCT for values of microwave power increasing from 100 to $1200 \mathrm{~W}$ through $10 \mathrm{~W}$ steps. ACCT current is almost constant from 0.4 to $6 \mathrm{~ms}$ for microwave power larger than $150 \mathrm{~W}$. 
microwave power larger than $150 \mathrm{~W}$. Moreover, rise and fall times are of the order of few hundreds of microseconds, much smaller than the pulse length. Several works show that plasma parameters reach steady state conditions in $\sim 0.6 \mathrm{~ms}$ after plasma switch on [30,31]. Therefore, for the purposes of this work, PS-ESS plasma can be assumed in steady state conditions during OES measurements. (3) During the OES experimental campaign on the PS-ESS proton source, it was not possible to measure the neutral and ion temperature. Yonesu et al. [32] performed measurements of neutral and ion temperature in experimental conditions very close to PS-ESS. They found a neutral temperature $T_{n} \sim 0.05 \mathrm{eV}$ and ion temperature $T_{i} \sim 0.4 \mathrm{eV}$ at $1 \times 10^{-3}$ mbar operative pressure and $2.5 \mathrm{~kW}$ microwave power. The value of neutral temperature has been confirmed also by measurements reported in Ref. [11]. In this work, we hypothesize that $T_{n}$ and $T_{i}$ assume similar values as given by the previous references. (4) Ion lifetime $\tau$ is assumed to be proportional to the square root of the ion mass. Indeed, $\tau$ is inversely proportional to the ambipolar diffusion coefficient, which, in turn, is inversely proportional to the square root of the ion mass (more information in Refs. [27,28,33]). Therefore, the following relation holds:

$$
\tau_{\mathrm{H}^{+}}: \tau_{\mathrm{H}_{2}^{+}}: \tau_{\mathrm{H}_{3}^{+}}=1: \sqrt{2}: \sqrt{3} .
$$

\section{MODEL DESCRIPTION}

The estimation of the beam parameters from plasma parameters has been performed through a three-stage procedure.

\section{A. Stage 1}

Neutral pressure is measured in the low-energy beam transport. However, if the hydrogen flow rate in the plasma chamber and the geometry of the proton source are known, it is possible to evaluate an average value of the neutral pressure inside the plasma chamber. Neutral pressure $P$ depends on the neutral density and temperature as follows:

$$
P=N_{\mathrm{H}} T_{n}+N_{\mathrm{H}_{2}} T_{n} .
$$

According to hypothesis (iii) of Sec. II B, we assume a constant neutral temperature, while the ratio $N_{\mathrm{H}} / N_{\mathrm{H}_{2}}$ is known from the OES measurements. This allows one to evaluate the absolute value of $N_{\mathrm{H}}$ and $N_{\mathrm{H}_{2}}$ for any experimental value of $N_{\mathrm{H}} / N_{\mathrm{H}_{2}}$.

\section{B. Stage 2}

In this stage, the following nonlinear balance equation system in the unknowns $n_{\mathrm{H}^{+}}, n_{\mathrm{H}_{2}^{+}}, n_{\mathrm{H}_{3}^{+}}$, and $\tau_{\mathrm{H}^{+}}$is solved:

$$
\begin{aligned}
& n_{e}=n_{\mathrm{H}^{+}}+n_{\mathrm{H}_{2}^{+}}+n_{\mathrm{H}_{3}^{+}}, \\
& \sum_{i} R_{\mathrm{H}^{+}}^{i}-\frac{n_{\mathrm{H}^{+}}}{\tau_{\mathrm{H}^{+}}}=0, \\
& \sum_{i} R_{\mathrm{H}_{2}^{+}}^{i}-\frac{n_{\mathrm{H}_{2}^{+}}}{\tau_{\mathrm{H}_{2}^{+}}}=\sum_{i} R_{\mathrm{H}_{2}^{+}}^{i}-\frac{n_{\mathrm{H}_{2}^{+}}}{\sqrt{2} \tau_{\mathrm{H}^{+}}}=0, \\
& \sum_{i} R_{\mathrm{H}_{3}^{+}}^{i}-\frac{n_{\mathrm{H}_{3}^{+}}}{\tau_{\mathrm{H}_{3}^{+}}}=\sum_{i} R_{\mathrm{H}_{3}^{+}}^{i}-\frac{n_{\mathrm{H}_{3}^{+}}}{\sqrt{3} \tau_{\mathrm{H}^{+}}}=0,
\end{aligned}
$$

$n_{\mathrm{H}^{+}}, n_{\mathrm{H}_{2}^{+}}$, and $n_{\mathrm{H}_{3}^{+}}$being the density of $\mathrm{H}^{+}, \mathrm{H}_{2}^{+}$, and $\mathrm{H}_{3}^{+}$, respectively, in plasma, and $\tau_{\mathrm{H}^{+}}, \tau_{\mathrm{H}_{2}^{+}}$, and $\tau_{\mathrm{H}_{3}^{+}}$are the confinement times for the three species. $\tau_{\mathrm{H}_{2}^{+}}$and $\tau_{\mathrm{H}_{3}^{+}}$have been replaced, respectively, with $\sqrt{2} \tau_{\mathrm{H}^{+}}$and $\sqrt{3} \tau_{\mathrm{H}^{+}}$ according to hypothesis (iv) of Sec. II B.

$R^{i}$ represents the volume creation or loss rate of the generic reactions $i$ for each ion species. In particular, $R^{i}$ is defined as

$$
R^{i}=n_{A} n_{B} Q^{i},
$$

where $n_{A}$ and $n_{B}$ are the densities of the interacting species and $Q^{i}$ is known as the reaction rate coefficient. For a Maxwellian distribution, $Q_{i}$ is only a function of $T_{e}$ [33]:

$$
\begin{aligned}
Q^{i}\left(T_{e}\right)= & \int_{0}^{\infty} \sigma_{i} f(v) v d v=6.7 \times 10^{7} \\
& \times T_{e}^{-3 / 2} \int_{0}^{\infty} E \sigma_{i} e^{-E / T_{e}} d E .
\end{aligned}
$$

In this work, we have considered 13 reactions having a cross section larger than $10^{-18} \mathrm{~cm}^{2}$ in the energy domain $0-100 \mathrm{eV}$ [34]. A list of the reactions is presented in Table I. Figure 5 shows the cross sections as a function of the electron temperature. In this picture, $\sigma_{10}$ is a constant, because it does not depend on the electron temperature but on the ion temperature, assumed to be a constant. $\sigma_{10}$ has

TABLE I. List of the cross sections taken into account for proton fraction estimation [34].

\begin{tabular}{lll}
\hline \hline & \multicolumn{1}{c}{ Reaction } & \multicolumn{1}{c}{ Process } \\
\hline$\sigma_{1}$ & $\mathrm{H}_{2}+e^{-} \rightarrow \mathrm{H}+\mathrm{H}+e^{-}$ & Dissociative excitation \\
$\sigma_{2}$ & $\mathrm{H}_{2}+e^{-} \rightarrow \mathrm{H}_{2}^{+}+2 e^{-}$ & Ionization \\
$\sigma_{3}$ & $\mathrm{H}_{2}+e^{-} \rightarrow \mathrm{H}^{+}+\mathrm{H}+2 e^{-}$ & Dissociative ionization \\
$\sigma_{4}$ & $\mathrm{H}_{2}^{+}+e^{-} \rightarrow \mathrm{H}^{+}+\mathrm{H}+e^{-}$ & Dissociative excitation \\
$\sigma_{5}$ & $\mathrm{H}_{2}^{+}+e^{-} \rightarrow 2 \mathrm{H}^{+}+2 e^{-}$ & Dissociative ionization \\
$\sigma_{6}$ & $\mathrm{H}+e^{-} \rightarrow \mathrm{H}^{+}+2 e^{-}$ & Ionization \\
$\sigma_{7}$ & $\mathrm{H}+e^{-} \rightarrow \mathrm{H}^{2 s}+e^{-}$ & Excitation to $\mathrm{H}^{2 s}$ state \\
$\sigma_{8}$ & $\mathrm{H}^{2 s}+e^{-} \rightarrow \mathrm{H}^{+}+2 e^{-}$ & Ionization from $\mathrm{H}^{2 s}$ state \\
$\sigma_{9}$ & $\mathrm{H}_{2}^{+}+e^{-} \rightarrow 2 \mathrm{H}$ & Dissociative recombination \\
$\sigma_{10}$ & $\mathrm{H}_{2}^{+}+\mathrm{H} \rightarrow \mathrm{H}_{3}^{+}+\mathrm{H}$ & Ion generation \\
$\sigma_{11}$ & $\mathrm{H}_{3}^{+}+e^{-} \rightarrow \mathrm{H}^{+}+2 \mathrm{H}+e^{-}$ & Dissociative excitation \\
$\sigma_{12}$ & $\mathrm{H}_{3}^{+}+e^{-} \rightarrow 3 \mathrm{H}$ & Dissociative recombination \\
\hline \hline
\end{tabular}




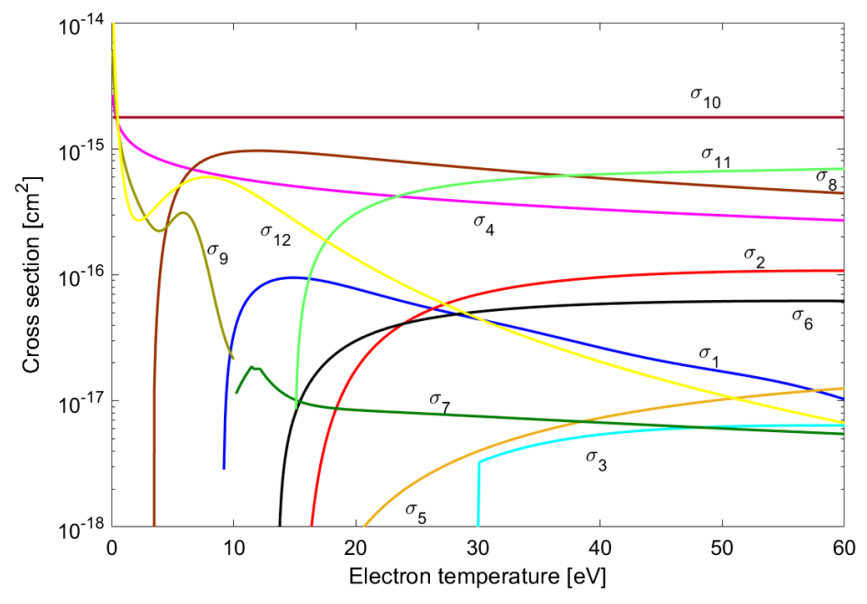

FIG. 5. Cross section of the 13 main reactions in a hydrogen plasma in the energy domain typical of MDIS [34].

been evaluated by replacing the electron temperature with the ion temperature in Eq. (7). Consequently, the solution of the nonlinear balance equation system $\left(n_{\mathrm{H}^{+}}, n_{\mathrm{H}_{2}^{+}}, n_{\mathrm{H}_{3}^{+}}\right.$, and $\tau_{\mathrm{H}^{+}}$) is a function of the input experimental parameters $n_{e}, T_{e}, N_{\mathrm{H}}$, and $N_{\mathrm{H}_{2}}$. Since the input parameters are affected by experimental errors, Eq. (5) has been solved within the whole interval $\left[n_{e} \pm \Delta n_{e}, T_{e} \pm \Delta T_{e}\right.$, $\left.N_{\mathrm{H}} / N_{\mathrm{H}_{2}} \pm \Delta\left(N_{\mathrm{H}} / N_{\mathrm{H}_{2}}\right)\right]$. This approach has allowed us to evaluate the error bars of the solution of Eq. (5) and, therefore, the error of the estimated beam parameters.

\section{Stage 3}

The evaluation of the ion density in the previous stage enables us to evaluate the overall beam intensity and the ion species fractions.

According to Eqs. (1) and (2), it is possible to estimate the extracted current for any ion species as

$$
I_{i}(z)=I_{i}(z=0)=\frac{1}{2} q \pi r^{2} n_{i} v_{\mathrm{Bohm}}^{i} .
$$

The total extracted current $I_{\text {extr }}$ is the sum of the partial currents:

$$
I_{\mathrm{extr}}=\sum_{i} I_{i}
$$

Finally, the ion species fractions are estimated as

$$
\begin{aligned}
& \mathrm{H}^{+}(\%)=\frac{I_{\mathrm{H}^{+}}}{I_{\mathrm{extr}}}, \\
& \mathrm{H}_{2}^{+}(\%)=\frac{I_{\mathrm{H}_{2}^{+}}}{I_{\mathrm{extr}}}, \\
& \mathrm{H}_{3}^{+}(\%)=\frac{I_{\mathrm{H}_{3}^{+}}}{I_{\mathrm{extr}}} .
\end{aligned}
$$

\section{COMPARISON BETWEEN EXPERIMENTAL RESULTS AND CODE ESTIMATION}

In this section, the extracted current and species fractions estimated by the semiempirical approach are compared with the experimental results obtained on the PS-ESS proton source. Extracted beam characterization and OES measurements have been performed in the same working conditions for a microwave power increasing from 150 to $1200 \mathrm{~W}$ and a neutral pressure $P=1 \times 10^{-3}$ mbar. Unfortunately, for microwave power higher than $800 \mathrm{~W}$, OES did not allow us to evaluate the $N_{\mathrm{H}} / N_{\mathrm{H}_{2}}$, due to a too small signal to noise ratio. For this reason, a complete set of OES plasma parameters to be used as input in Eq. (5) was available only in the range $150-800 \mathrm{~W}$.

The measured extracted current has been evaluated from data in Fig. 4. Pulse stability $\Delta I_{\text {extr }}^{p} / \bar{I}_{\text {extr }}$ during the $6 \mathrm{~ms}$ interval is always in the range $1 \%-4 \%$ for any value of microwave power, while pulse to pulse stability $\Delta I_{\text {extr }}^{p 2 p} / \bar{I}_{\text {extr }}$ does not overcome $2 \%$. Therefore, the error on $I_{\text {extr }}$ has been evaluated as $\sqrt{\left(\Delta I_{\text {extr }}^{p} / \bar{I}_{\text {extr }}\right)^{2}+\left(\Delta I_{\text {extr }}^{p 2 p} / \bar{I}_{\text {extr }}\right)^{2}}$.

The Doppler-shift unit gives a measurement of the species fraction averaged in $4 \mathrm{~s}$, a time much higher than the duration of a single pulse. The comparison between different measurements, performed at constant microwave power, shows a very high parameter stability and repeatability $(<1 \%)$. Therefore, the error on the measurement of species fraction is negligible for the scope of the present paper.

Figure 6 shows the comparison between the total extracted current measured by ACCT and the value of total current estimated from the semiempirical approach. The value of extracted current is predicted with good accuracy in most of the experimental conditions. Figure 7 shows the comparison between the species fraction

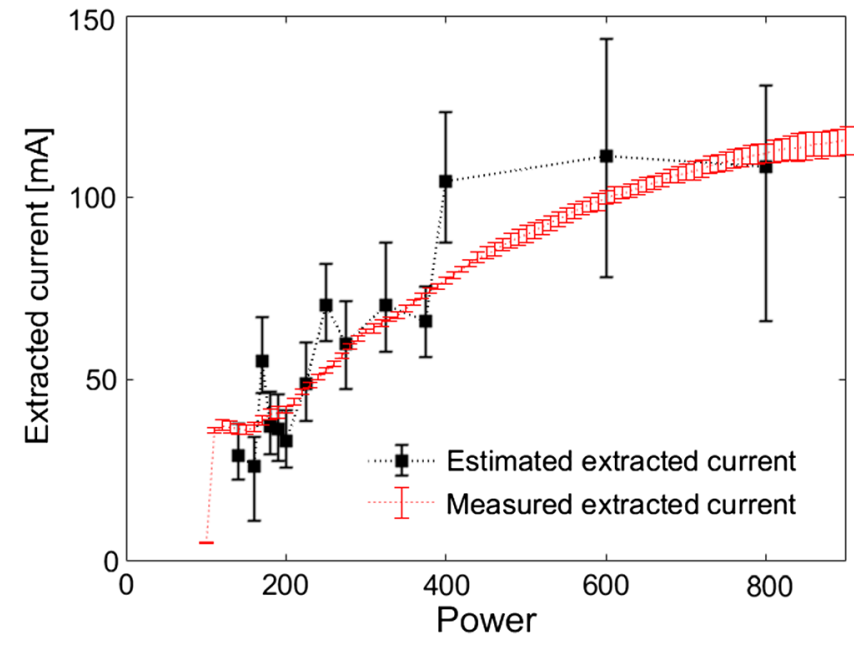

FIG. 6. Comparison between the experimental value of extracted current measured by ACCT and total current predicted by means of Eqs. (8) and (9). 


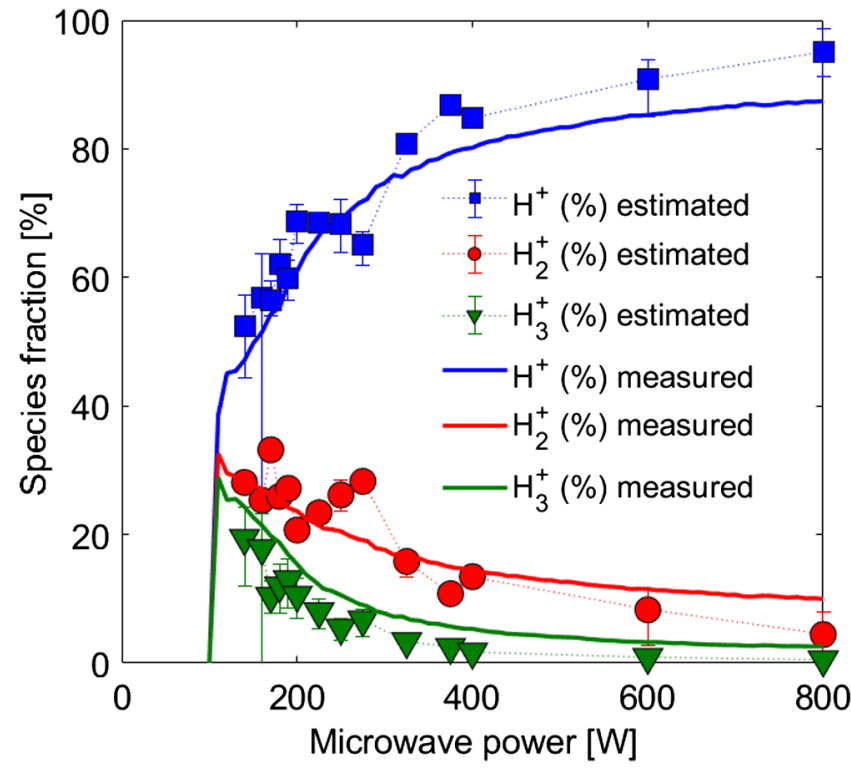

FIG. 7. Comparison between experimental values of the species fraction measured by Doppler-shift measurement unit and the values predicted by code.

measured by the Doppler-shift measurement unit and the species fraction estimated by the semiempirical approach. Also in this case, a good prediction capability is found for a large part of the experimental values. The results are less accurate at higher power. A possible reason is the decrease of the plasma electron temperature at high power. This causes the $\mathrm{H}_{2}^{+}$and $\mathrm{H}_{3}^{+}$generation and destruction reactions (related to cross sections $\sigma_{4}, \sigma_{9}$, and $\sigma_{12}$ ) to become more and more relevant with respect to the other reactions. $\mathrm{H}_{2}^{+}$and $\mathrm{H}_{3}^{+}$generation and destruction reactions depend on $T_{n}$ and $T_{i}$, here assumed to be constants. Consequently, any systematic error concerning the value of $T_{n}$ and $T_{i}$ may play a role and negatively affect the results.

The solution of the semiempirical balance equation system gives, together with the beam species currents, also an estimation of the proton lifetime $\tau_{\mathrm{H}^{+}}$. To support this result, we made a theoretical estimation of the confinement time expected in the PS-ESS proton source.

In MDIS, the magnetic field of proton sources is almost flat and does not confine particles. So, the confinement time is mainly determined by the peculiar diffusion mechanisms of magnetized plasmas [1].

In the presence of a magnetic field, particle diffusion takes place via interaction with a self-generated electric field, which modifies opportunely electron and ion loss fluxes to maintain the plasma quasineutrality [28].

Two different diffusion mechanisms can arise: (i) Simon diffusion.-A surplus of positive loss fluxes across the magnetic field is matched by a surplus of negative loss fluxes along the magnetic field. Surface currents, called the Simon currents, guarantee overall charge neutralization [35]. (ii) Ambipolar diffusion.-If Simon currents cannot flow due to insulated walls, positive and negative loss fluxes have to match along any diffusion direction, i.e., either along and across the magnetic field $[28,36]$. It has been experimentally proven that ambipolar diffusion optimizes MDIS performances [27].More information about plasma diffusion in ion sources can be found in Refs. [28,36].

In PS-ESS, the injection and extraction sides of the plasma chamber are constituted by two boron nitride disks, avoiding Simon currents to flow. Therefore, the ambipolar diffusion can be considered as the preferential diffusion mechanism [27]. Furthermore, according to Refs. [27,28], ambipolar diffusion across the magnetic field is negligible with respect to ambipolar diffusion along the magnetic field. Consequently, the proton confinement time in PS-ESS can be evaluated as the average time for protons to diffuse from the position where they are generated to the extraction electrode along a flux tube of length $L$ and radius $r$ under ambipolar diffusion conditions.

In particular, for an ion generated at a distance $x$ from extraction, confinement time $\tau(x)$ is [28]

$$
\tau(x)=\frac{x^{2}}{2 D_{\|}^{a m b}},
$$

where $D_{\|}^{a m b}$ is the ambipolar diffusion coefficient along the magnetic field [28]. The evaluation of the mean confinement time requires one to average over all the possible positions where ions are generated. This treatment is beyond the scope of the present paper. However, the two extreme cases are considered. In the first case, we hypothesize that ions are generated homogeneously within the tube flux. In such a case, the average theoretical confinement time $\tau_{\mathrm{H}^{+}}^{\text {theorl }}$ is

$$
\bar{\tau}_{\mathrm{H}^{+}}^{\text {theorl }}=\int_{0}^{L} \frac{x^{2} n_{i} \pi r^{2} d x}{2 D_{\|}^{a m b}} \cdot \frac{1}{n_{i} \pi r^{2} L}=\frac{L^{2}}{6 D_{\|}^{a m b}},
$$

where $n_{i} \pi r^{2} d x$ is the number of ions in a circular slab of width $d x$ and radius $r$ placed at distance $x$ from the walls and $n_{i} \pi r^{2} L$ is the total number of particles in the tube flux.

In the second case, we hypothesize that ions are generated mainly at the microwave injection, where the electric field is larger and an ECR layer is placed [37]. In such a case, the confinement time is

$$
\bar{\tau}_{\mathrm{H}^{+}}^{\mathrm{theor} 2}=\frac{L^{2}}{2 D_{\|}^{a m b}}
$$

It is reasonable that the real confinement $\bar{\tau}$ time is included in the range $\bar{\tau}_{\mathrm{H}^{+}}^{\text {theor1 }}<\bar{\tau}<\bar{\tau}_{\mathrm{H}^{+}}^{\text {theor2 }}$.

$D_{\|}^{a m b}$ can be expressed as a function of the ion and electron mobility coefficients $\mu_{i}$ and $\mu_{e}$, respectively, and of 


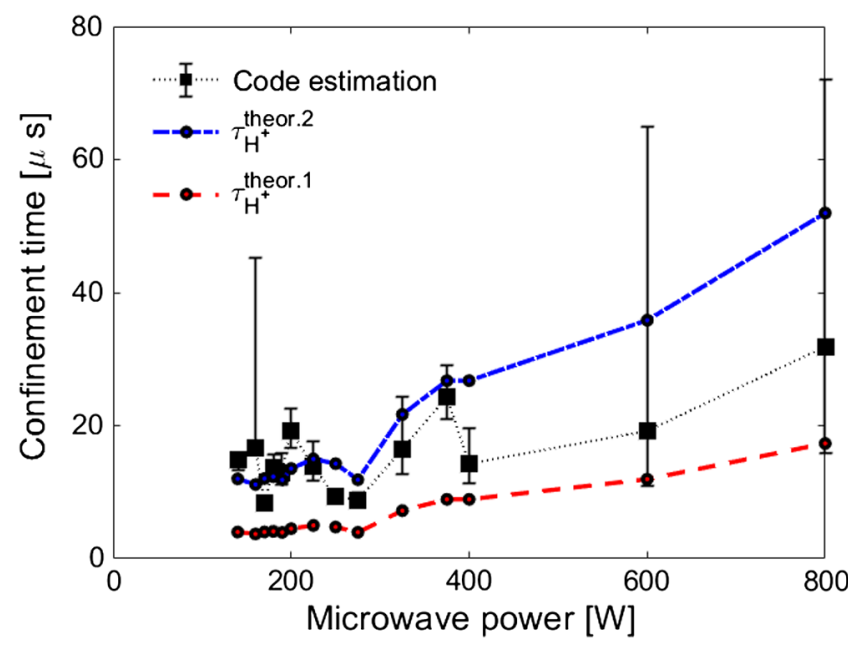

FIG. 8. Proton confinement time $\bar{\tau}_{\mathrm{H}^{+}}$estimated by the semiempirical approach is supported by the comparison with the two borderline values of theoretical confinement time. $\bar{\tau}_{\mathrm{H}^{+}}^{\text {theor1 }}<\bar{\tau}_{\mathrm{H}^{+}}<$ $\bar{\tau}_{\mathrm{H}^{+}}^{\text {theor2 }}$ in the largest part of cases.

the electron and ion diffusion coefficients along magnetic field $D_{\| i}$ and $D_{\| e}$ :

$$
D_{\|}^{a m b}=\frac{\mu_{i} D_{\| e}+\mu_{e} D_{\| i}}{\mu_{i}+\mu_{e}} .
$$

In proton sources, the largest part of the collisions in plasmas are long-range Coulomb scatters and ion-neutral collisions (the latter include elastic and charge exchange collisions) [1]. Consequently, $\mu_{i}, \mu_{e}, D_{\| e}$, and $D_{\| i}$ can be written as [1]

$$
\begin{aligned}
\mu_{e} & =\frac{1}{M_{e}\left(\nu_{e e}^{\text {Spitz }}+\nu_{e i}^{\text {Spitz }}\right)}, \\
\mu_{i} & =\frac{1}{M_{i}\left(\nu_{i i}^{\text {Spitz }}+\nu_{e i}^{\text {Spitz }}+\nu_{\text {in }}\right)}, \\
D_{\| e} & =\frac{T_{e}}{M_{e}\left(\nu_{e e}^{\text {Spitz }}+\nu_{e i}^{\text {Spitz }}\right)}, \\
D_{\| i} & =\frac{T_{i}}{M_{i}\left(\nu_{i i}^{\text {Spitz }}+\nu_{e i}^{\text {Spitz }}+\nu_{\text {in }}\right)},
\end{aligned}
$$

where $\nu_{e e}^{\text {Spitz }}, \nu_{i i}^{\text {Spitz }}$, and $\nu_{e i}^{\text {Spitz }}$ are the Spitzer collision frequencies for electron-electron, ion-ion, and electronion collisions, respectively; $\nu_{\text {in }}$ is the ion-neutral collision frequency. $\nu_{e e}^{\text {Spitz }}, \nu_{i i}^{\text {Spitz }}$, and $\nu_{e i}^{\text {Spitz }}$ are functions of $n_{e}, T_{e}$, and $T_{i}$ and can be easily evaluated from the literature $[1,28]$. Figure 8 compares the code estimation $\bar{\tau}_{\mathrm{H}^{+}}$obtained as a solution of the balance equation system with $\bar{\tau}_{\mathrm{H}^{+}}^{\text {theor1 }}$ and $\bar{\tau}_{\mathrm{H}^{+}}^{\text {theor2 }}$ obtained by Eqs. (12) and (13).

$\bar{\tau}_{\mathrm{H}^{+}}$is between $\bar{\tau}_{\mathrm{H}^{+}}^{\text {theorl }}$ and $\bar{\tau}_{\mathrm{H}^{+}}^{\text {theor } 2}$ for the largest part of the values.
The good agreement between the results obtained by the two different approaches supports the value of confinement time estimated by the semiempirical approach. $\bar{\tau}_{\mathrm{H}^{+}}$ increases slightly from $\sim 10 \mu \mathrm{s}$ at $180 \mathrm{~W}$ to $\sim 30_{-15}^{+35} \mu \mathrm{s}$ at $800 \mathrm{~W}$. The percentage error increases also with microwave power, and it is of the order of $100 \%$ at 600 and $800 \mathrm{~W}$. As in the case of the extracted current, the error is caused by the error in the evaluation of the electron temperature by OES [26].

\section{CONCLUSIONS AND PERSPECTIVES}

This work introduces a semiempirical strategy aimed at the estimation of the beam features from plasma parameters. The balance equation system in a proton source has been reduced and simplified by introducing experimental plasma parameters as input parameters. In this work, plasma parameters have been measured by OES, which allows an accurate and noninvasive measurement of $n_{e}, T_{e}$, and $n_{\mathrm{H}} / n_{\mathrm{H}_{2}}$. In particular, the solution of the balance equation system has allowed estimating the total extracted current, the species fractions, and the confinement time under a series of assumptions and hypothesis discussed in Secs. II A and II B. The comparison between the values predicted by the semiempirical approach and the experimental values measured on the PS-ESS source has shown a surprising agreement, although PS-ESS works in pulsed waves. This result can be considered as a validation of the strategy that underlies the semiempirical approach.

A value of the proton confinement time, slightly increasing from $\sim 10$ to $\sim 30 \mu$ s with microwave power, is also found as a solution of the balance equation system. It has been compared with two theoretical values of confinement time in ambipolar diffusion conditions. Also in this case, the theoretical approach confirms the results of the semiempirical approach.

Therefore, we can state that the semiempirical approach allows one to estimate with good accuracy the features of the beam generated by a MDIS proton source. Further improvements can be obtained by including experimental neutral and ion temperature within the input parameter set. Measurements of $T_{n}$ and $T_{i}$ can be performed by OES by using a spectrometer with a FWHM of the order of $10 \mathrm{pm}$ [11] (PS-ESS measurements were carried out with a $1 \mathrm{~nm}$ FWHM spectrometer [26]).

The semiempirical approach requires also to be accurately tested in continuous wave MDIS proton sources. Indeed, steady state working conditions allow one to reduce the number of approximations in Secs. II A and II B.

In perspective, the semiempirical approach, applied in synergy with OES diagnostics, could boost the $R \& D$ in the field of MDIS proton sources. It could allow a real-time, cost-effective, and small footprint diagnostics of the beam properties, also in the absence of an extraction system, and lead to an overall decrease of the beam commissioning time in proton accelerators. 


\section{ACKNOWLEDGMENTS}

The support of the Ministero dell'Istruzione, dell'Università e della Ricerca through the ESS-Miur experiment is gratefully acknowledged. The contribution and suggestions of L. Neri, L. Celona, G. D'Agostino, and M. Mazzaglia have been essential for the preparation of the present work.

[1] R. Geller, Electron Cyclotron Resonance Ion Sources and ECR plasmas (Institute of Physics, Bristol, 1996).

[2] R. Miracoli, L. Celona, G. Castro, D. Mascali, S. Gammino, D. Lanaia, R. Di Giugno, T. Serafino, and G. Ciavola, Characterization of the versatile ion source and possible applications as injector for future projects, Rev. Sci. Instrum. 83, 02A305 (2012).

[3] J. M. Lagniel et al., Status and new developments of the high intensity electron cyclotron resonance source light ion continuous wave, and pulsed mode, Rev. Sci. Instrum. 71, 830 (2000).

[4] G. Castro, G. Torrisi, L. Celona, D. Mascali, L. Neri, G. Sorbello, O. Leonardi, G. Patti, G. Castorina, and S. Gammino, A new $\mathrm{H}_{2}{ }^{+}$source: Conceptual study and experimental test of an upgraded version of the VISVersatile ion source, Rev. Sci. Instrum. 87, 083303 (2016).

[5] Y. Xu, S. Peng, H. Ren, J. Zhao, J. Chen, A. Zhang, T. Zhang, Z. Guo, and J. Chen, High current $\mathrm{H}_{2}{ }^{+}$and $\mathrm{H}_{3}{ }^{+}$beam generation by pulsed $2.45 \mathrm{GHz}$ electron cyclotron resonance ion source, Rev. Sci. Instrum. 85, 02 A943 (2014).

[6] G. Castro et al., Enhanced production of $\mathrm{He}^{+}$from the Versatile Ion Source (VIS) in off-resonance configuration, Rev. Sci. Instrum. 85, 096109 (2014).

[7] G. Castro, Multi-diagnostics investigation of an ECR plasma confined in a linear axis-symmetric trap, Nuovo Cimento Soc. Ital. Fis. C 42, 224 (2019).

[8] U. Fantz, Basics of plasma spectroscopy, Plasma Sources Sci. Technol. 15, S137 (2006).

[9] U. Fantz, H. Falter, P. Franzen, D. Wünderlich, M. Berger, A. Lorenz, W. Kraus, P. McNeely, R. Riedl, and E. Speth, Spectroscopy - a powerful diagnostic tool in source development, Nucl. Fusion 46, S297 (2006).

[10] R. Kronholm, T. Kalvas, H. Koivisto, J. Laulainen, M. Marttinen, M. Sakildien, and O. Tarvainen, Spectroscopic study of ion temperature in minimum-B ECRIS plasma, Plasma Sources Sci. Technol. 28, 075006 (2019).

[11] S. Briefi, D. Rauner, and U. Fantz, Determination of the rotational population of $\mathrm{H} 2$ and $\mathrm{D} 2$ including high-N states in low temperature plasmas via the Fulcher- $\alpha$ transition, J. Quant. Spectrosc. Radiat. Transfer 187, 135 (2017).

[12] H. R. Griem, Principles of Plasma Spectroscopy (Cambridge University Press, Cambridge, England, 1997).

[13] T. Fujimoto, Plasma Spectroscopy (Clarendon, Oxford, 2004).

[14] W. Yang, S. N. Averkin, A. V. Khrabrov, I. D. Kaganovich, Y.-N. Wang, S. Aleiferis, and P. Svarnas, Benchmarking and validation of global model code for negative hydrogen ion sources, Phys. Plasmas 25, 113509 (2018).
[15] R. Zorat, J. Goss, D. Boilson, and D. Vender, Global model of a radiofrequency $\mathrm{H}_{2} \mathrm{H} 2$ plasma in DENISE, Plasma Sources Sci. Technol. 9, 161 (2000).

[16] F. Gaboriau and J.P. Boeuf, Chemical kinetics of low pressure high density hydrogen plasmas: Application to negative ion sources for ITER, Plasma Sources Sci. Technol. 23, 065032 (2014).

[17] W. Yang, H Li, F. Gao, and Y. N. Wang, Hybrid simulations of solenoidal radio-frequency inductively coupled hydrogen discharges at low pressures, Phys. Plasmas 23, 123517 (2016).

[18] C. M. Samuell and C.S. Corr, Low-pressure hydrogen plasmas explored using a global model, Plasma Sources Sci. Technol. 25, 015014 (2016).

[19] R. Huh, N. K. Kim, B. K. Jung, K. J. Chung, Y. S. Hwang, and G.H. Kim, Global model analysis of negative ion generation in low-pressure inductively coupled hydrogen plasmas with bi-Maxwellian electron energy distributions, Phys. Plasmas 22, 033506 (2015).

[20] W. Yang, H. Li, F. Gao, and Y. N. Wang, A global model study of the population dynamics of molecular hydrogen and the generation of negative hydrogen ions in lowpressure ICP discharge with an expansion region: effects of EEPF, Plasma Sources Sci. Technol. 27, 075006 (2018).

[21] C. D. Child, Discharge from hot CaO, Phys. Rev. 32, 492 (1911).

[22] R. Garoby et al., The european spallation source design, Phys. Scr. 93, 014001 (2018).

[23] L. Celona et al., High intensity proton source and LEBT for the European spallation source, AIP Conf. Proc. 2011, 020019 (2018).

[24] L. Neri et al., Beam commission of the high intensity proton source developed at INFN-LNS for the European Spallation Source, J. Phys. Conf. Ser. 874, 012037 (2017).

[25] D. Wünderlich, M. Giacomin, R. Ritz, and U. Fantz, Yacora on the Web: Online collisional radiative models for plasmas containing $\mathrm{H}, \mathrm{H}_{2}$ or $\mathrm{He}$, J. Quant. Spectrosc. Radiat. Transfer 240, 106695 (2020).

[26] M. Mazzaglia, G. Castro, D. Mascali, L. Celona, L. Neri, G. Torrisi, S. Gammino, R. Reitano, and E. Naselli, Improvement of the characterization of the proton source for the European Spallation Source by means of optical emission spectroscopy, Phys. Rev. Accel. Beams 22, 053401 (2019).

[27] G. Castro, D. Mascali, R. Miracoli, L. Celona, and S. Gammino, Diffusion processes in microwave discharge ion source and consequences on the upgrade of existing ion sources, Rev. Sci. Instrum. 90, 023301 (2019).

[28] A. Liebermann and A.J. Liechtemberg, Principles of Plasma Discharges and Materials Processing (Wiley, New York, 2005).

[29] A. Guthrie and R. K. Wakerling, The Characteristics of Electrical Discharges in Magnetic Fields (McGraw-Hill, New York, 1949).

[30] W. Wu et al., Status of high current $\mathrm{H}_{2}{ }^{+}$and $\mathrm{H}_{3}{ }^{+}$ion sources, Rev. Sci. Instrum. 90, 101501 (2019).

[31] Y. Xu, S. Peng, H. Ren, J. Zhao, J. Chen, A. Zhang, T. Zhang, Z. Guo, and J. Chen, High current $\mathrm{H}_{2}{ }^{+}$and $\mathrm{H}_{3}{ }^{+}$beam generation by pulsed $2.45 \mathrm{GHz}$ electron cyclotron resonance ion source, Rev. Sci. Instrum. 85, 02 A943 (2014). 
[32] A. Yonesu, S. Shinohara, Y. Yamashiro, and Y. Kawai, Ion and neutral temperatures in an electron cyclotron resonance plasma, Thin Solid Films 390, 208 (2001).

[33] H. Zhang, Ion Sources (Springer, New York, 2001).

[34] R. K. Janev, D. Reiter, and U. Samm, Collision processes in low-temperature hydrogen plasmas Report Forschungszentrum Jülich JUEL 4105 (2003), http://juser.fz-juelich .de/record/38224/files/Juel_4105_Reiter.pdf.
[35] A. Simon, Ambipolar diffusion in a magnetic field, Phys. Rev. 98, 317 (1955).

[36] A. G. Drentje, Simon short circuit effect in ECRIS, Rev. Sci Instrum. 73, 516 (2002).

[37] L. Neri, G. Castro, G. Torrisi, A. Galatà, D. Mascali, L. Celona, and S. Gammino, Recent progress in plasma modelling at INFN-LNS, Rev. Sci. Instrum. 87, 02A505 (2016). 